\title{
Property of Regenerating Serotonin Fibers in the Hippocampus of Human Migration Disorders Model
}

\author{
Shuichi UEDA, Ayuka EHARA and Hideki OHMOMO \\ Department of Histology and Neurobiology, Dokkyo Medical University School of Medicine, Mibu, Tochigi 321-0293, Japan
}

\begin{abstract}
Individual mood and mental conditions exert a great influence on one's own kansei. Abnormality or dysfunction of the 5-HT neuron system in the developing and/or adult brain is closely associated with their conditions. Thus, the 5-HT neuron system may play an important role in the neuronal mechanisms underlying kansei. Interestingly, previous studies have shown that heterotopic clusters in the hippocampus (hippocampal heterotopia), deriving from neocortical neurons, after prenatally treated with methylazoxymethanol acetate in rat (MAM rat), exhibit abundant 5-HT innervation. After neonatal intracisternal 5,7-dihydroxytryptamine (DHT) injection, these 5-HT fibers degenerate and disappear throughout the forebrain, and then regenerating 5-HT fibers densely innervate in the hippocampal heterotopia. The 5-HT fiber system in the hippocampal heterotopia of MAM rat provides useful experimental models for study the plasticity of human migration disorder. In the present study, to evaluate the properties of regenerating 5-HT fibers in the hippocampal heterotopia of MAM rats, we examined the origin of these projections by combined retrograde transport and immunohistochemical methods. Prenatal exposure to MAM resulted in the formation of hippocampal heterotopia in the dorsal hippocampus. Regenerating 5-HT fibers formed a dense innervation within the hippocampal heterotopia after neonatal DHT injection. These projections appeared to arise mainly from 5-HT neurons in the median raphe nucleus, with a small portion from 5-HT neurons in the dorsal raphe nucleus. These findings suggest a specific profile of regenerating 5-HT fibers, providing the new insights for serotonergic plasticity.

Keywords: Serotonin, Regeneration, 5,7-dihydroxytryptamine, Methylazoxymethanol, Hippocampus
\end{abstract}

\section{INTRODUCTION}

Serotonin (5-hydroxytryptamine, 5-HT) is an important neurotransmitter and neuromodulator. Abnormality or dysfunction of the 5-HT neuron system in the developing and/or adult brain is closely associated with psychological and mental illnesses such as autism, depression, anxiety disorders and schizophrenia [1]. Therefore, 5-HT is considered a key molecule in the regulation of mind or mood functions of the brain [2]. Such disorders may be interpreted as perturbation of the neuronal mechanisms underlying kansei [3]. Thus, the 5-HT neuron system may play an important role in the neuronal mechanisms underlying kansei.

In the brain, cell bodies of the 5-HT neuron system are located in raphe nuclei of the brainstem, and their fibers are distributed broadly throughout the brain and spinal cord [4]. Serotonergic fibers innervating the forebrain region originate predominantly from the dorsal raphe nucleus (DRN) and median raphe nucleus (MRN) [5]. Fibers originating from the MRN distribute predominantly to midline and para-midline brain structures, whereas fibers from the DRN primarily project laterally [6, 7]. On the basis of the different projection areas, the DRN and MRN appear to provide different contributions to brain functions.
In addition to these global projections, the 5-HT neuron system has a vast capacity for regenerative change for several types of brain lesions [4, 8, 9]. However, the reinnervation pattern of regenerating 5-HT fibers depends on the target area of injured axons, the type of injury and the age at which injury occurs. For example, the hippocampus of adult rats shows variable density of regenerating 5-HT fibers at 8 to 12 weeks after intraventricular 5,7dihydroxytryptamine (DHT) injection, whereas neonatal intracisternal DHT injection induces long-lasting 5-HT depletion, without evidence of hippocampal regeneration [10-12].

Methylazoxymethanol acetate (MAM) is an antimitotic agent that interferes with nucleic acid methylation [13]. Injection of MAM into rats on embryonic day 15 (E15) substantially reduces the size of the cerebral cortex and also results in ectopic neurons in the hippocampal formation (hippocampal heterotopia), which causes micrencephaly (small cerebrum) $[14,15]$. The MAM-induced micrencephalic rats (MAM rats) have been used as a model to study human migration disorders [13-15]. Children with migration disorders exhibit significant clinical problems, such as drug resistant epilepsy, mental retardation, autism and uncontrollable mood disorders. Immunohistochemical study of surgically resected tissue from children with cryptogenic epilepsies has revealed that abnormal (heterotopic) tissue receives serotonergic hyper-innervation. It has been 
speculated that this abnormal innervation plays some role in the compensatory mechanisms for neuronal dysfunction in cortical dysplasia [16]. Thus, this model would provide new insights into the mechanisms of enhanced serotonergic plasticity as well as a promising clue for therapeutic intervention of psychological and mental illnesses with disturbed kansei.

The brains of MAM rats show significant neurochemical as well as neuroanatomical changes [17, 18]. The hippocampal 5-HT content in MAM rats is greater than that in control rats $[17,19]$. Our previous study demonstrated that the regeneration of 5-HT fibers in MAM rats occurred from 4 to 5 weeks after neonatal intracisternal DHT injection, and these fibers extend into the hippocampus [19]. At 3 months after injection, the area of hippocampal heterotopia shows a normal density of regenerating 5-HT fibers, whereas other parts of the hippocampus show lower density of 5-HT fibers [19]. Thus, it has been suggested that the area of hippocampal heterotopia exerts a trophic effect for regenerating 5-HT fibers in the developing MAM rat. However, the origins of these regenerating 5-HT fibers have not been demonstrated.

In the present study, we investigated the origin of regenerating 5-HT fibers in the area of hippocampal heterotopia in MAM rats using combined methods of retrograde tracing and immunohistochemistry.

\section{MATERIALS AND METHODS}

\subsection{Animals and experimental conditions}

All procedures in the present study were approved by the Animal Care and Use Committee, Dokkyo Medical University School of Medicine. Male and female SpragueDawley rats were obtained from Japan Charles River Laboratories (Tsukuba, Japan) and bred under controlled conditions of temperature and humidity with a 12-h dark/ light cycle and continuous access to food and water. Rats were mated overnight, and those with a vaginal plug on the following day were classified as embryonic day 0 (E0) and separated from the males. Plug checks were conducted at 10:00 AM. A schema of the experimental schedule is shown in Figure 1.

MAM (MidWest Research Institute, Kansas City, MO) was diluted in saline and injected intraperitoneally (i.p.) at a dose of $25 \mathrm{mg} / \mathrm{kg}$ body weight at 10:00 AM in E15 rats as described previously [19]. For the DHT injection, 3-day-old (P3d) rat pups were initially injected with desipramine (20 mg/kg i.p.; Sigma-Aldrich, St. Louis, MO,), a catecholamine uptake inhibitor. One hour later, the half of these pups were injected intracisternally with DHT (50 mg free base in $10 \mathrm{ml}$ saline with $0.1 \%$ ascorbic acid volume of saline). The pups were returned to the animal cages and foster dams and weaned at 4 weeks of age. At 14 weeks after birth, the rats from each experimental group were examined. Animals were divided into 4 groups (Fig. 1): saline treated (E15) and saline injected (P3d) rats (control group), saline treated (E15) and DHT injected (P3d) rats (DHT group), MAM treated (E15) and saline injected (P3d) rats (MAM group), and MAM treated (E15) and DHT injected $(\mathrm{P} 3 \mathrm{~d})$ rats $(\mathrm{MAM}+\mathrm{DHT}$ group).

\subsection{Biochemical measurements}

Tissue concentrations (pM/mg wet tissue) of 5-HT and its metabolite, 5-hydroxyindoleacetic acid (5-HIAA), in the hippocampus were measured by high-performance liquid chromatography (HPLC). The detailed methods were previously described $[17,20]$. In brief, rats (6 per group) were decapitated, and brains were removed immediately, washed in ice-cold $0.1 \mathrm{M}$ phosphate buffered saline (PBS, pH 7.4), rapidly dissected and frozen in liquid nitrogen. The hippocampus was weighed, homogenized at least in 10 volumes of $0.1 \mathrm{M}$ perchloric acid containing $0.1 \mathrm{mM} \mathrm{Na}$-ethylenediaminetetraacetic acid, and filtered. The filtered homogenate was centrifuged, and the supernatant was analyzed.

\subsection{Tissue preparation and immunohistochemistry}

Under deep anesthesia, rats were perfused transcardially with physiological saline, followed by $500 \mathrm{ml}$ of $4 \%$ paraformaldehyde plus $0.2 \%$ picric acid in $0.1 \mathrm{M} \mathrm{PB}$. Brains were removed immediately and post-fixed in the same fixative for $48 \mathrm{~h}$ at $4^{\circ} \mathrm{C}$, then immersed in $20 \%$ sucrose solution for $48 \mathrm{~h}$. Brains were then frozen, and serial cryostat sections $(30 \mu \mathrm{m})$ were collected in $0.1 \mathrm{M}$ PBS containing $0.3 \%$ Triton-X100.

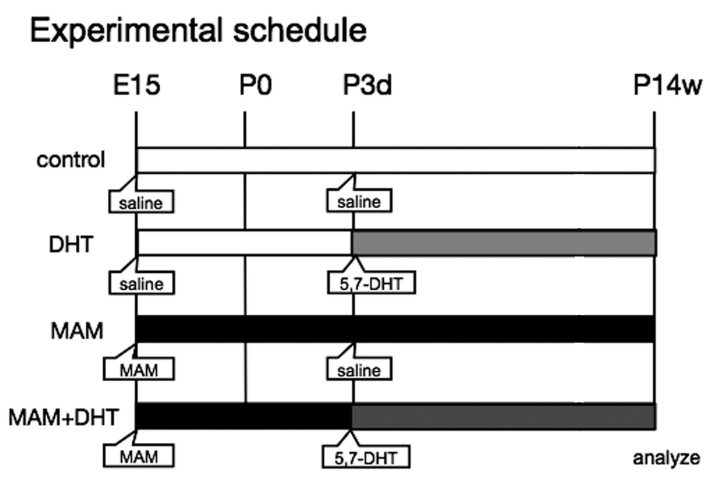

Figure 1: Experimental design of the present study. Rats were assigned to one of four groups: control, DHT, MAM and MAM+DHT. MAM $(25 \mathrm{mg} / \mathrm{kg})$ was injected intraperitoneally into pregnant rats at E15. At P3d, half of the rats were treated with intracisternal DHT injection. At P14w, rats were examined by HPLC and immunohistochemistry. 
Sections were incubated in $1 \% \mathrm{H}_{2} \mathrm{O}_{2}$ solution and then treated with $1.5 \%$ normal horse serum for $1 \mathrm{~h}$ at room temperature to prevent non-specific staining. Sections were then incubated with goat anti-5-HT antibody (1:2000 - 10,000; DiaSorin, Stillwater, MN or Abcam, Cambridge, MA) for 7 days at $4^{\circ} \mathrm{C}$. After several times rinses in $\mathrm{PBS}$, sections were processed for avidin-biotin peroxidase immunostaining with biotinylated anti-goat IgG and an ABC kit (Vector Labs, Inc., Burlingame, CA). To compare the number of 5-HT neurons in the DRN and MRN, the total number of 5HT-labeled neurons was counted in 12 brainstem sections ( $150 \mu \mathrm{m}$ distance between sections) including the DRN and MRN. Results were assessed statistically compared with Student two-tailed t-test (significant level of 0.05).

\subsection{Fluoro-Gold injection and double-labeling}

Five rats from the MAM $(n=3)$ and MAM+DHT $(n=2)$ groups received injection of a 5\% Fluoro-Gold (FG) solution (Fluorochrome, Denver, $\mathrm{CO}$ ) into the bilateral dorsal hippocampus. After 7 days, injected rats were perfused transcardially with physiological saline, followed by $500 \mathrm{ml}$ of $4 \%$ paraformaldehyde in $0.1 \mathrm{M}$ PB. Thick $(30 \mu \mathrm{m})$ free-floating sections were cut with a cryostat. After a wash, the sections were further incubated with the antibody against 5-HT and then with species-specific Alexa Fluor 568 secondary antibody (Molecular Probes, Eugene, OR). After several washes, the sections were mounted onto glass slides. Images were acquired with a FV500 confocal laser-scanning microscope (Olympus, Tokyo, Japan).

All statistical data are expressed as mean \pm standard error of the mean (SEM).

\section{RESULTS}

\subsection{General neuroanatomical findings and HPLC analysis}

Similar to our previous MAM experiments [18-21], rats in the MAM and MAM+DHT groups showed a thin cortex and hippocampal heterotopias in area CA1. In Nissl-stained sections, no morphological differences were observed between the DHT group and the Control group (data not shown). In brief, hippocampal 5-HT and 5-HIAA concentrations increased in the MAM group, but decreased both the DHT and MAM+DHT groups (data not shown).

\subsection{Immunohistochemical analysis}

Cells immunoreactive for 5-HT were distributed throughout the brainstem in all experimental groups, and were concentrated in the DRN and MRN (Fig. 2). The DHT group showed a consistent reduction in 5-HTimmunoreactive cells in the DRN and MRN compared to the Control group, with decreases of $88 \%$ and $46.6 \%$, respectively (Figs. 2A-D, 3). There were no significant differences in the number of 5-HT-immunoreactive cells in the DRN and MRN between the MAM and Control groups (Figs. 2A, B, E, F, 3). The MAM+DHT group also showed a considerable decrease in 5-HT-immunoreactive cells in the DRN (83.9\% cell loss compared to the control group, $\mathrm{p}<0.001)$. However, the reduction was limited to the MRN (23.2\% cell loss compared to the control group, no statistical significance) (Figs. 2E-H, 3).

The 5-HT-immunoreactive fibers were distributed widely throughout the brain in the Control and MAM groups. Similar to results of our previous study [19], immunohistochemical analysis showed hyperinnervation by 5 -HT fibers in the hippocampus in the MAM group. Confirming neurochemical data, DHT injection induced a
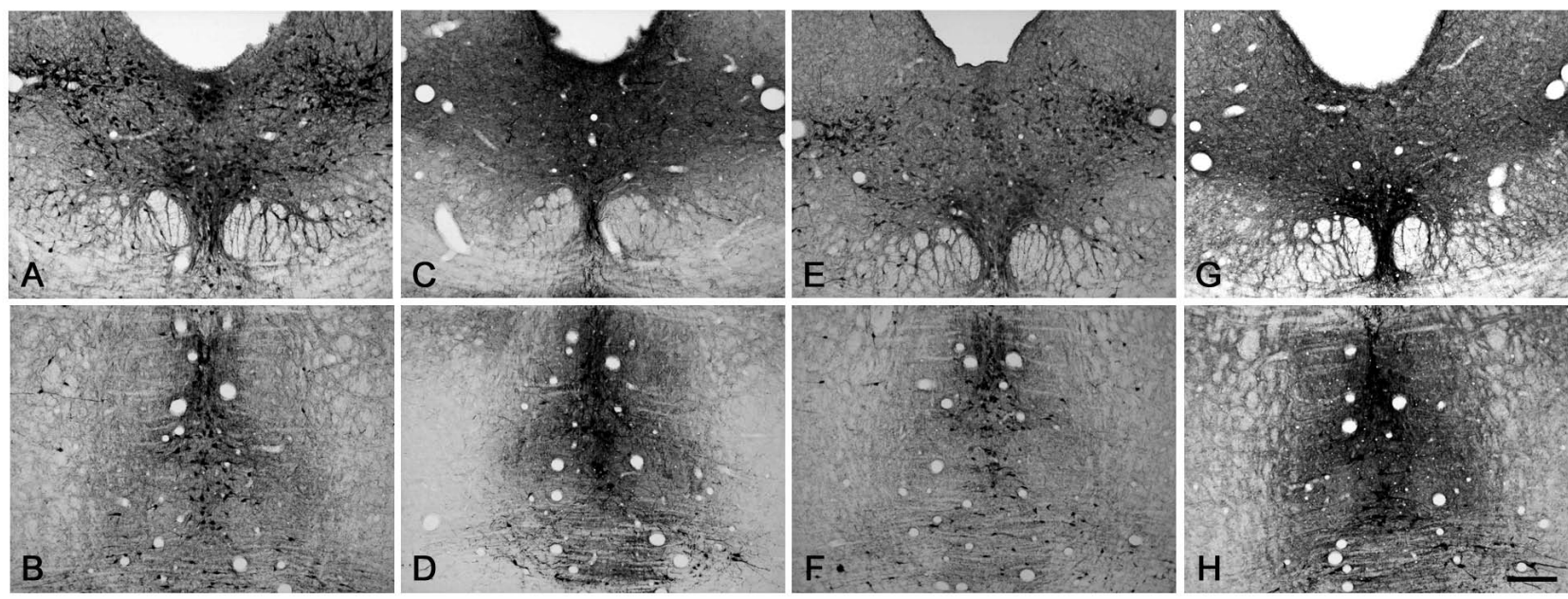

Figure 2: Photomicrographs of 5-HT immunohistochemical staining in the DRN (A, C, E, G) and MRN (B, D, F, H) of control (A, B), DHT (C, D), MAM (E, F) and MAM+DHT (G, H) rats. Scale bar, $250 \mu \mathrm{m}$. 


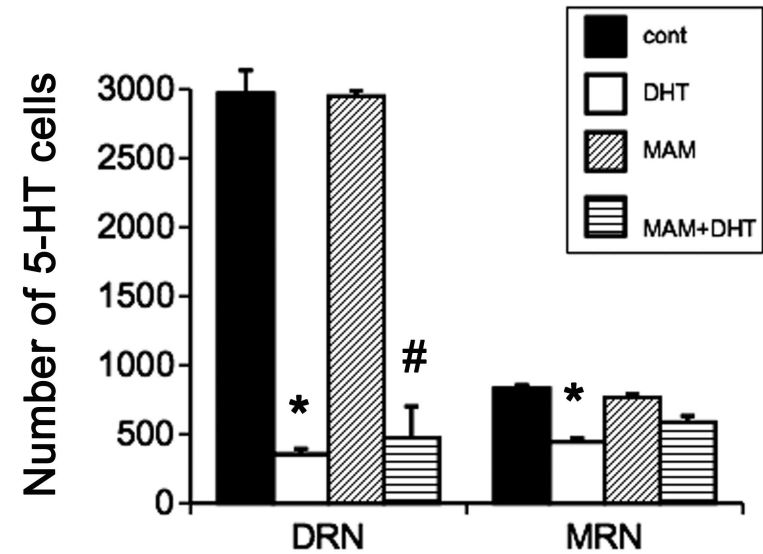

Figure 3: Quantification of 5-HT-immunoreactive neurons in the DRN and MRN of 14-week-old control, DHT, MAM and MAM+DHT rats.

$* \mathrm{p}<0.05$ vs Control group;

$\# \mathrm{p}<0.05$ vs MAM group.

significant reduction of these immunoreactive fibers in both the DHT group and the MAM+DHT group (data not shown). In the MAM+DHT group, the area of hippocampal heterotopia was endowed with large numbers of 5-HT fibers, whereas the density of 5-HT fibers in the other parts of the hippocampus remained unchanged (Fig. 4).

\subsection{Analysis of the origin of 5-HT fibers}

After DHT injection on P3d, most of these fibers degenerated and almost completely disappeared [19]. After several weeks, regenerating 5-HT fibers extended terminal fields into the hippocampus and the area of hippocampal heterotopia in the MAM+DHT group at 14 weeks of age was densely reinnervated [19]. In subsequent experiments, we examined the origin of 5-HT fibers in the area of hippocampal heterotopia in the MAM and MAM+DHT groups.

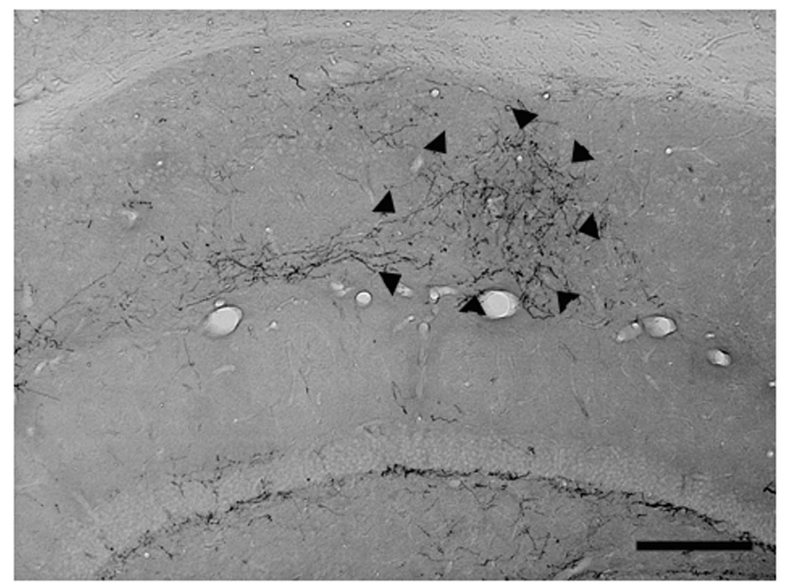

Figure 4: Photomicrograph of a coronal section from the dorsal hippocampus of a 14-week-old MAM+ DHT rat stained for 5-HT immuno-histochemistry. The area of hippocampal heterotopia (arrowheads) is densely innervated by 5 -HT fibers. Scale bar, $500 \mu \mathrm{m}$.
The retrograde tracer FG was injected bilaterally into the dorsal hippocampus, where hippocampal heterotopia was evident (Fig. 5A), and FG-labeled cells were observed throughout the brain including the cerebral cortex, thalamus, hypothalamus and brainstem. Colocalization data for FG-label and 5-HT signal-label in the DRN and MRN of the MAM and MAM+DHT groups (Fig. 5B) are summarized in Figure 6. The numbers of double-labeled cells in the DRN and MRN were $183.0 \pm 10.4$ and 242.3 \pm 5.2 , respectively, in the MAM group and $37.3 \pm 8.9$ and $63.6 \pm 14.6$, respectively, in the MAM+DHT group.

\section{DISCUSSION}

MAM rats serve as a useful model for investigating genetic and epigenetic neuronal dysgenesis in the brain [13]. Hyperinnervation by the 5-HT neuron system in hypoplastic cortex and hippocampus of MAM rats has been well investigated by neurochemical $[13,17,18,20]$

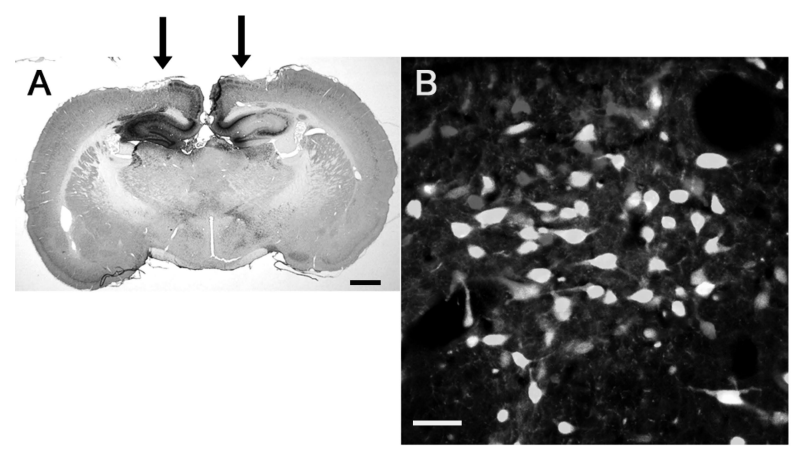

Figure 5: A. Low-power photomicrograph of a representative FG injection site in the bilateral dorsal hippocampus. Arrows indicate injection sites. Scale bar, $1 \mathrm{~mm}$. B. High-power photomicrograph of FG and 5-HT immuno-fluorescence histochemistry in the MRN of a MAM rat. Double-labeled cells show intense signal. Scale bar, $50 \mu \mathrm{m}$.
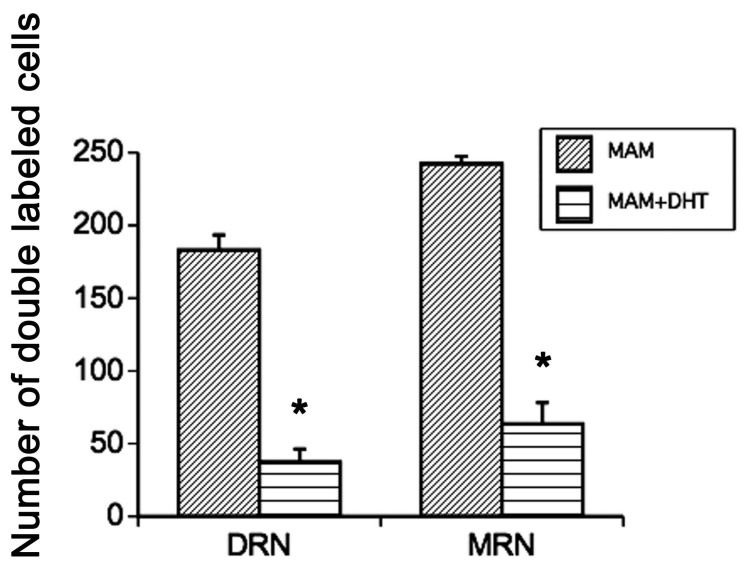

Figure 6: Quantification of cells double-labeled for FG and 5-HT in the DRN and MRN of MAM and MAM+DHT rats. $* \mathrm{p}<0.01$ vs MAM group. 
and immunohistochemical methods $[18,19]$. In our previous study, the hippocampal heterotopia is densely innervated by regenerated 5-HT fibers, however, we could not visualize individual 5-HT neurons in the DRN and MRN of these rats because of high background staining in these areas [19]. Thus, we could not assess the number of 5-HT neurons in the DRN and MRN in the DHT and MAM+DHT groups. In the present study, we used the 5-HT antibody different from which had used in our previous study and we further visualized individual 5-HT neurons in the DRN and MRN. A previous study reported that prenatal (E15) MAM treated to Wistar rats reduces the number of DRN and MRN 5-HT neurons at P35d [22]. However, they neither provided quantitative data nor compared MAM-treated rats to saline-treated rats. In the present study, we provided quantitative evidence for no significant difference in the numbers of 5-HT neurons in the DRN and MRN between MAM- and saline-injected rats. After DHT injection, the number of 5-HT neurons in the DRN in the MAM+DHT group was significantly reduced. However, there was no statistical difference in the number of 5-HT neurons in the MRN between the MAM and MAM+DHT groups. The present data from MAM rats confirm the evidence from normal rats, that 5-HT neurons in the DRN are more vulnerable to neonatal DHT lesion than those in the MRN [10] and further demonstrate that 5-HT neurons in the MRN of MAM rats are more resistant to DHT than those of untreated rats.

In intact rats, the hippocampus receives dense 5-HT innervation from both the DRN and MRN. However, 5-HT fibers from the DRN and MRN show a differently distribution within the dentate gyrus; fibers from MRN project to the stratum radiatum of area CA3 and the region between the stratum radiatum and the stratum lacunosummoleculare of area CA1. In the dentate gyrus of intact rats, 5-HT fibers arising from the DRN project preferentially to the molecular layer, whereas 5-HT fibers from the MRN project mainly to the hilar regions, forming a dense plexus adjacent to the granule cell layer [5, 7]. In MAM rats, most 5-HT fibers in the hippocampal formation degenerate after intracisternal DHT injection, with surviving 5-HT fibers then sprouting and reorganizing within the hippocampal formation. The connections of 5-HT fibers between the area of hippocampal heterotopia and the plexuses in the stratum lacunosum-moleculare or hilar regions suggest the sprouting profiles of 5-HT fibers [19]. Thus, two distinct entrances of regenerating 5-HT fibers within the area of hippocampal heterotopia have been speculated, via the stratum lacunosum-moleculare and hilar routes. In the present study, the number of retrogradely FG-labeled 5-HT positive cells in the MRN was approximately two times greater than that in the DRN of the MAM+DHT group. This indicates that regenerating 5-HT fibers originated mainly from the MRN, supporting our previous hypothesis that regenerating 5-HT fibers in the area of hippocampal heterotopia are of MRN origin [19].

MAM rats are reportedly highly susceptible to seizure in response to several epileptogenic agents, and heterotopic tissue in these rats shows hyperexcitability, owing to the formation of abnormal connection between surrounding areas [15]. Heterotopic tissue in MAM rats intrinsically receives abundant GABAergic innervation, similar to that in human dysplasia, and this GABAergic system forms inhibitory synapses with neurons in the heterotopic tissue. Thus, the activity of the inhibitory system against seizures is enhanced in heterotopic tissue [23]. It is well known that an increased extracellular 5-HT level inhibits seizures, whereas 5-HT depletion results in a decreased seizure threshold [24]. Excessive 5-HT release from hyperinnervating 5-HT fibers in the developing cortex of MAM rats has been demonstrated by the microdialysis method [18]. Thus, in addition to the intrinsic GABAergic system, hyperinnervating 5-HT fibers in the area of hippocampal heterotopia may show marked resistance to seizure activity [21]. In addition, 5-HT has been proposed as an important trophic or maintenance factor for other neuronal systems during development and after brain damage $[20,25,26]$. The synaptic density of non-serotonergic neurons in the developing and adult brain is regulated by the extracellular 5-HT level [26-28]. Taken together, it appears likely that synaptic formation within the area of hippocampal heterotopia is maintained by 5 -HT hyper-innervation.

In conclusion, the results of the present study showed that regenerating 5-HT fibers from the MRN preferentially extended the terminal field into the area of hippocampal heterotopia. This hippocampal heterotopia may exert trophic effects for developing and regenerating 5-HT fibers. We propose that the area of hippocampal heterotopia in MAM rats might serve as a good model to study plasticity of 5-HT innervation in vivo, providing the new insights for serotonergic plasticity underlying the neuronal mechanisms of kansei.

\section{REFERENCES}

1. D.S. Charney, E.J. Nesstler (eds.); Neurobiology of Mental Illness, 2nd ed. Oxford University Press, New York; (2004).

2. T. Shiga, K. Suda, H. Yoshida, S. Nakamura, M. Natsume, M. Yoshikawa, K. Senzaki; Serotonin: A key regulator for the development of brain and 
mind, Kansei Engineering International; 6(2), pp.1924 (2006).

3. M. Sugiyama, A. Ina, S. Hisano; Effects of prenatal stress on early postnatal development of glutamatergic neurons in the mouse brain, Kansei Engineering Internatinal; 6(2), pp.35-42 (2006).

4. B.L. Jacobs, E.C. Azmitia; Structure and function of the brain serotonin system, Physiological Reviews; 72(1), pp.165-229 (1992).

5. E.C. Azmitia, M. Segal; An autoradiographic analysis of the differential ascending projections of the dorsal and median raphe nuclei in the rat, Journal of Comparative Neurology; 179(3), pp.641-668 (1978).

6. L.P. Morin, LE. Meyer-Bernstein; The ascending serotonergic system in the hamster: Comparison with projections of the dorsal and median raphe nuclei, Neuroscience; 91(1), pp.81-105 (1999).

7. R.P. Vertes, W.J. Fortin, A.M. Crane; Projections of the median raphe nucleus in the rat, Journal of Comparative Neurology; 407(4), pp.555-582 (1999).

8. A. Bjorklund, U. Stenevi; Regeneration of monoaminergic and cholinergic neurons in the mammal-ian central nervous system, Physiological Reviews; 59(1), pp.62-100 (1979).

9. S. Ueda, Y. Sano, M. Kawata; Collateral sprouting of serotonergic fibers in the cingulate cortex and the septum following cortical-hippocampal lesions, Brain Research; 556(2), pp.329-332 (1991).

10. A. Towel, G.R. Breese, R.A. Mueller, S. Coyle, J.M. Lauder; Early postnatal administration of 5,7-DHT: Effects on serotonergic neurons and terminals, Brain Research; 310(1), pp.67-75 (1984).

11. S. Ueda, M. Kawata; Regeneration of serotonergic immunoreactive fibers in the brain of 5,6-dihydroxytryptamine treated rat, Journal of Brain Research; 35(1), pp.158-180 (1994).

12. S. Ueda, S. Sakakibara, K. Yoshimoto; Effect of long-lasting serotonin depletion on environmental enrichment-induced neurogenesis in adult rat hippocampus and spatial learning, Neuroscience; 135(2), pp.395-402 (2005).

13. M.V. Johnston, J.T. Coyle; Cytotoxic lesions and the development of transmitter system; Trends in Neurosciences; 5, pp.153-182 (1982).

14. F. Catabeni, M.D. Lica; Developmental models of brain dysfunctions induced by targeted cellular ablations with methylazoxymethanol, Physiological Reviews; 77(1), pp.199-215(1997).

15. N. Chevassus-Au-Louis, A. Rafiki, I. Jorquera, Y. Ben-Ari, A. Represa; Neocortex in the hippocampus: An anatomical and functional study of CA1 heterotopias after prenatal treatment with methylazoxymathanol in rats, Journal of Comparative Neurology; 394(4), pp.520-536 (1998).

16. S. Trottier, B. Evrard, J.P. Vignal, J.M. Scarabin, P. Chauvel; The serotonergic innervation of the cerebral cortex in man and its changes in focal cortical dysplasia, Epilepsy Research; 25(2), pp.79-106 (1996).

17. G. Jonsson, H. Hallman; Effects of prenatal methylazoxymethanol treatment on the development of central monoaminergic neurons, Developmental Brain Research; 2(4), pp.513-530 (1982).

18. S. Ueda, A. Nishimura, T. Kusuki, Y. Takeuchi, K. Yoshimoto; Delayed 5-HT release in the developing cortex of micrencephalic rats, NeuroReport; 10(6), pp.1215-1219 (1999).

19. A. Nakamura,T. Kadowaki,S. Sakakibara,K. Yoshimoto, K. Hirata, S. Ueda; Regeneration of 5-HT fibers in hippocampal heterotopia of methylazoxymethanolinduced micrencephalic rats after neonatal 5, 7-DHT injection, Anatomical Science International; 85(1), pp.38-45 (2010).

20. S. Ueda, K. Yoshimoto, T. Kadowaki, K. Hirata, S. Sakakibara; Improved learning in micrencephalic rats, Congenital Anomalies; 50(1), pp.58-63 (2010).

21. Y. Watabe, K. Yoshimoto, M. Eguchi, S. Ueda; Degeneration of monoaminergic fibers in the aged micrencephalic rat, Neuroscience Letters; 385(1), pp.82-86 (2005).

22. A. Funahashi, M. Inoue, H. Yamamura; Developmental alteration of serotonin neurons in the raphe nucleus of rats with methylazoxymethanol-induced micrencephaly, Acta Neuropathologica; 85(1), pp.3138 (1992).

23. M.E. Calcagonotto, M.F. Paredes, S.C. Baraban; Heterotopic neurons with altered inhibitory synaptic function in an animal model of malformation-associated epilepsy, Journal of Neuroscience; 22(17), pp.75967605 (2002).

24. G. Bagdy, V. Kecskemeti, P. Riba, R. Jakus; Serotonin and epilepsy, Journal of Neurochemistry; 100(4), pp.857-873 (2007).

25. N. Okado, M. Narita, N. Narita; A biogenic aminesynapse mechanism for mental retardation and developmental disabilities, Brain and Development; 23(suppl 1), pp.S11-S15 (2001).

26. M. Matsukawa, K. Nakadate, I. Ishihara, N. Okado; Synaptic loss following depletion of noradrenaline and/or serotonin in the rat visual cortex: A quantitative electron microscopic study, Neuroscience; 122(3), pp.627-635 (2003)

27. M. Emerit, M. Riad, M. Hamon; Trophic effects of neurotransmitters during brain maturation, Biology 
of the Neonate; 62(4), pp.193-201 (1992).

28. C.C. Wilson, K.M. Faber, J.H. Haring; Serotonin regulates synaptic connections in the dentate molecular layer of adult rats via 5-HT1a receptors: Evidence for a glial mechanism, Brain Research; 782(1-2), pp.235239 (1998).

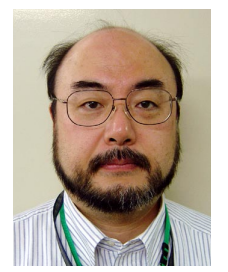

\section{Shuichi UEDA}

Shuichi UEDA is a Professor and Head of the Department of Histology and Neurobiology, Dokkyo Medical University School of Medicine since 1995. He received B.S. at Nihon University, School of Medicine in 1981, and Ph.D. at Kyoto Prefectural University of Medicine in 1986. His research is focused on neuronal mechanisms controlling emotion.

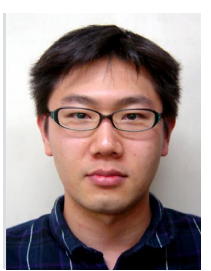

Hideki OHMOMO

Hideki OHMOMO is an Assistant Professor of the Department of Histology and Neurobiology, Dokkyo Medical University School of Medicine, Tochigi Japan, since 2006. He received Ph.D. degree from the University of Tsukuba, Japan, in 2012. His research interest is neuronal mechanisms of aggressive behavior and their control.

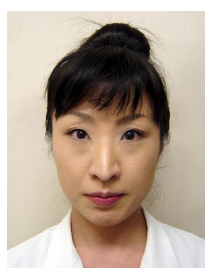

Ayuka EHARA

Ayuka EHARA is an Assistant Professor of Department of Histology and Neurobiology, Dokkyo Medical University School of Medicine, Tochigi Japan, since 2008. She received her B.S. (2003) in Life Science, Ochanomizu University, and Ph.D (2008) in Kansei, Behavioral and Brain Sciences,

University of Tsukuba. Her research interest is Brain Neuroscience. 